\title{
A symmetric splitting method for rigid body dynamics II
}

\author{
E. CELLEDONI*† and N. SÄFSTRÖM†
}

Keywords: Rigid bodies, symmetric methods, symplectic methods, Jacobi elliptic functions

It has been known since the time of Jacobi that the solution to the free rigid body Euler equations of motion is given in terms of a certain type of elliptic functions. Using the Arithmetic-Geometric mean algorithm, Abramowitz \& Stegun (1992), these functions can be calculated efficiently and accurately. The overall approach yields a faster and more accurate numerical solution to the Euler equations compared to standard numericul ODE and symplectic solvers.

In this paper we investigate the possibility of extending this approach to the case of rigid bodies subject to external forces. By using a splitting strategy similar to the one proposed in Reich (1996), we decompose the vector field of our problem in a free rigid body (FRB) problem and another completely integrable vector field. We apply the method to the simulation of the heavy top.

\section{Introduction}

The study of numerical discretizations of Hamiltonian systems which preserve one or several of the geometric features of the continuous equations, has been quite popular in the last few decades, Hairer et al. (2002). Leimkuhler \& Reich (2004): Symplectic methods are suited for the numerical solution of Hamiltonian problems. The energy is almost conserved along the numerical flow computed by a symplectic method. with essentially no accumulation of errors in time, Hairer et al. (2002). Moreover these methods show long-term stability of the integration. Symplectic Runge-Kutta methods are designed to preserve the symplectic structure of canonical Hamiltonian problems,

$$
\dot{q}=\frac{\partial H(q, p)}{\partial p}, \quad \dot{p}=-\frac{\partial H(q, p)}{\partial q} .
$$

If the Hamiltonian problem considered is a holonomically constrained Hamiltonian system, (i.e. an algebraic constraint of the type $g(q)=0$ is also present), symplectic integration cannot be achieved by the straightforward application of symplectic methods, the use of appropriate partitioned Runge-Kutta methods is required, Hairer et al. (2002). A efficient way to address constrained Hamiltonian problems is, when possible, to split the constrained Hamiltonian vector field in a sum of two or more constrained Hamiltonian vector fields which can be separately integrated. The flows are then composed together to form the final symplectic numerical solution.

*Corresponding author.

†Department of Mathematical Sciences. NTNU. Trondheim. Norway.

IIAn early version of this paper was presented at SIMS 2005, the 46th Conference on Simulation and Modeling, Trondheim, Norway, October 13-14, 2005. 
A similar situation arises when the Hamiltonian problem is not given in the canonical form (1), Hairer et al. (2002). This is the case of the Euler equations describing the motion of a free rigid body (FRB),

$$
\begin{aligned}
& I_{1} \dot{\omega}_{1}=\left(I_{2}-I_{3}\right) \omega_{2} \omega_{3}, \\
& I_{2} \dot{\omega}_{2}=\left(I_{3}-I_{1}\right) \omega_{3} \omega_{1}, \\
& I_{3} \dot{\omega}_{3}=\left(I_{1}-I_{2}\right) \omega_{1} \omega_{2},
\end{aligned}
$$

where $I_{1}, I_{2}$ and $I_{3}$ are the principal moments of inertia. These equations are completely integrable. Energy and angular momentum are preserved along the solution, this means that for all times the two quantities

$$
\begin{aligned}
E & =I_{1}(1)_{1}^{2}+I_{2}\left(\omega_{2}^{2}+I_{3}\left(\omega_{3}^{2},\right.\right. \\
G^{2} & =I_{1}^{2}\left(\omega_{1}^{2}+I_{2}^{2}\left(\omega_{2}^{2}+I_{3}^{2}()_{3}^{2},\right.\right.
\end{aligned}
$$

are constant, (here $E$ is the energy and $G^{2}$ is the total angular momentum). There is also a non canonical symplectic structure (Lie-Poisson structure) preserved by the flow of (2), Leimkuhler \& Reich (2004).

By using the two constants of motion it is possible to derive the solution of the equations expressed in terms of Jacobi elliptic functions. Consider

$$
\begin{array}{ll}
a_{1}^{2}=2 E I_{3}-G^{2}, & a_{3}^{2}=G^{2}-2 E I_{1}, \\
b_{1}^{2}=I_{2}\left(I_{3}-I_{2}\right), & b_{3}^{2}=I_{2}\left(I_{2}-I_{1}\right),
\end{array}
$$

and assume $b_{3} / a_{3} \leq b_{1} / a_{1}$ (we will have a similar situation if $b_{3} / a_{3} \geq b_{1} / a_{1}$ ), the solutions of the Euler equations are

$$
\omega_{1}=\frac{a_{1} \mathrm{cn} u}{\sqrt{I_{1}\left(I_{3}-I_{1}\right)}}, \quad \omega_{2}=\frac{a_{1} \operatorname{sn} u}{b_{1}}, \quad \omega_{3}=\frac{a_{3} \mathrm{dn} u}{\sqrt{I_{3}\left(I_{3}-I_{1}\right)}},
$$

where the Jacobi elliptic functions $\mathrm{cn}$, sn and $\mathrm{dn}$, are defined by

$$
\operatorname{cn} u=\cos \varphi, \quad \operatorname{sn} u=\sin \varphi, \quad \operatorname{dn} u=\sqrt{1-k^{2} \sin ^{2} \varphi} .
$$

Here the amplitude $\varphi$ is given implicitly as the solution of the equation

$$
F\left(\varphi \mid k^{2}\right)=u(t), \quad u(t)=\lambda(t-\tau)
$$

where

$$
F\left(\varphi \mid k^{2}\right):=\int_{0}^{\varphi} \frac{d \theta}{\sqrt{1-k^{2} \sin ^{2} \theta}}
$$

is an elliptic integral of the first kind with modulus $k$. We have

$$
k=\frac{b_{3} a_{1}}{b_{1} a_{3}}, \quad \lambda=\frac{b_{1} a_{3}}{I_{2} \sqrt{l_{1} I_{3}}},
$$

and $\tau$ is a constant of integration that is used to satisfy the initial conditions.

The simulation of rigid body motion is interesting for applications in robotics, structural mechanics, and molecular dynamics, Geradin \& Cardona (2001), Leimkuhler \& Reich (2004). The formulae (5) for the exact solution of the Euler equations can be turned into a numerical method by using efficient numerical approximations of the Jacobi elliptic functions. In this paper we show how this approach is very competitive 
and can be further applied to problems of rigid bodies subject to external forces. We also refer to Bucher (2003) and Mitchell (2000) for related literature.

Symplectic integration methods for the Euler equations have been constructed by various authors, Ge \& Marsden (1988), Moser \& Veselov (1991), Lewis \& Simo (1994), see also Lrimkuhler \& Reich (2004) and references therein. In spite of being a source of insight, many of these methods cannot be straightforwardly generalized to the broader class of non canonical Hamiltonian problems, thus their use is limited to the numerical approximation of the Euler equations. However some of these integrators have successfully been applied in the simulation of rigid body dynamics. This is for instance the case in Reich (1996), Dullweber et al. (1997).

Recently McLachlan and Reich have, independently, proposed a splitting method for the rigid body equations McLachlan (1993), Reich (1996). In this method the right hand side of (2) is split in the following three terms,

$$
f_{1}((1))=\left[\begin{array}{c}
0 \\
I_{3}(1)_{3}(1)_{1} \\
-I_{2}(1)_{1}(1)_{2}
\end{array}\right], \quad f_{2}((1))=\left[\begin{array}{c}
-I_{3}(1)_{2}(1)_{3} \\
0 \\
I_{1}(1)_{1}(1)_{2}
\end{array}\right], f_{3}((1))=\left[\begin{array}{c}
I_{2}\left(\omega _ { 2 } \left(\omega_{3}\right.\right. \\
-I_{1}\left(\omega_{3} \omega_{1}\right. \\
0
\end{array}\right] .
$$

Each of the three vector fields is Hamiltonian with respect to the rigid body Poisson bracket Marsden \& Ratiu (1994), p. 8, and defines differential equations which is easy to integrate exactly. The appropriate composition of the corresponding flows produces a symplectic approximation of the problem. There is numerical evidence showing that this Lie-Poisson method is very competitive compared to most of the known and previously proposed strategies of symplectic integration of the Euler equations. Celledoni \& Owren (2003), McLachlan \& Zanna (2005). For this reason we will use this method as a comparison method in our numerical experiments.

Accurate, symplectic, energy and momentum preserving approximations for the solution of the Euler equations. have been recently addressed in McLachlan \& Zanna (2005). Our numerical tests show that such approximations can be easily achieved by using the exact solution and computing (6) to machine accuracy.

To solve problems modelled by rigid bodies subject to external forces, in Reich (1996), a symplectic method based on a splitting of the vector field into a FRB problem and another Hamiltonian vector field hats been considered. We will briefly recall this approach in the citse of the heavy top in section 4 . In the original approach of Reich (1996) the author uses the splitting method (8) 10 solve the FRB problem. In this paper we instead use the formulac (5) and compute the elliptic functions (6) to machine accuracy by the Arithmetic-Geometric mean algorithm. The overall splitting is symmetric, but not symplectic. However, in many of the considered numerical experiments, the new method presents better conservation of energy and better behavior of the numerical solution then the symplectic integrator of Reich (1996) and Dullweber at al. (1997).

Some technical issues for the implementation of this approach are discussed in section 3. In section 4 we report some numerical experiments comparing the proposed approach to the symplectic splitting of Reich (1996). Dullweber of al. (1997).

\section{A splitting method for the heavy top}

Efficient integrators for the free rigid body can be used in connection with splitting methods in the numerical approximation of more complex rigid body dynamics. We 
with the initial condition $\pi\left(t_{j}\right)=\pi^{(j)}$, computed at the previous time step. For this purpose we use the MATLAB standard function ellipj, which returns the values of

$$
\text { sn } u=\sin \varphi, \quad \text { cn } u=\cos \varphi, \quad \text { dn } u=\sqrt{1-k^{2} \sin ^{2} \varphi},
$$

for a given input $u=\lambda(t-\tau)$, with $t=\left(t_{j+1 / 2}, t_{j+1}\right)^{T}$ and $m=k^{2}$. Here $k$ and $\lambda$ are given constants, except for the sign (se section 1), and $\tau$ must be determined appropriately to ensure that the initial condition $\omega\left(t_{j}\right)=\omega^{(j)}$ is satisfied. To this end, we first find the amplitude $\varphi^{(j)} \in[0,2 \pi]$, which is uniquely determined from the equations

$$
\omega_{1}\left(t_{j}\right)=\frac{a_{1} \cos \varphi^{(j)}}{\sqrt{I_{1}\left(I_{3}-I_{1}\right)}}, \quad \omega_{2}\left(t_{j}\right)=\frac{a_{1} \sin \varphi^{(j)}}{b_{1}} .
$$

Furthermore, from the sign of $\omega_{3}\left(t_{j}\right)$,

$$
\omega_{3}\left(t_{j}\right)=\frac{a_{3} \sqrt{1-k^{2} \sin ^{2} \varphi^{(j)}}}{\sqrt{I_{3}\left(I_{3}-I_{1}\right)}},
$$

we determine the sign of the constants $a_{3}$ and $\lambda$. Since $u\left(t_{j}\right)$ must satisfy (7), we obtain that $\tau$

$$
\tau=t_{j}-\frac{1}{\lambda} F\left(\varphi^{(j)} \mid k^{2}\right)
$$

where

$$
F\left(\varphi^{(j)} \mid k^{2}\right)=\int_{0}^{\varphi^{(j)}} \frac{d \theta}{\sqrt{1-k^{2} \sin ^{2} \theta}} .
$$

The latter integral can be computed to machine accuracy using the method of Arithmetic-Geometric Mean, briefly described below.

Consider the sequence $\left\{\varphi_{n}^{(j)}\right\}_{n=0,1, \ldots, \varphi_{n+1}^{(j)}}>\varphi_{n}^{(j)}$, defined by

$$
\tan \left(\varphi_{n+1}^{(j)}-\varphi_{n}^{(j)}\right)=\frac{b_{n}}{a_{n}} \tan \left(\varphi_{n}^{(j)}\right), \quad \varphi_{0}^{(j)}=\varphi^{(j)}
$$

where $a_{n}, b_{n}$ are given by the Arithmetic-Geometric Mean series, Abramowitz \& Stegun (1992), i.e.

$$
a_{n+1}=\frac{a_{n}+b_{n}}{2}, b_{n+1}=\sqrt{a_{n} b_{n}}, c_{n+1}=\frac{a_{n}-b_{n}}{2} .
$$

Taking the initial values

$$
a_{0}=l, b_{0}=\sqrt{1-k^{2}}, c_{0}=k
$$

one can show that

$$
F\left(\varphi^{(j)} \mid k^{2}\right)=\lim _{n \rightarrow \infty} \frac{\varphi_{n}^{(j)}}{2^{n} a_{n}} .
$$

As the Geometric-Arithmetic Mean series converges quadratically, one obtains very accurate approximations of $F\left(e^{(j)} \mid k^{2}\right)$ in few recursion steps. In our implementation we terminate the iteration when $c_{N}$ is less than machine epsilon.

\section{Numerical experiments}

The splitting method proposed in this paper is compared with the symplectic method of Dullweber et al. (1997) and Reich (1996) which we denote in short by MR, and with 
the classical fourth order Runge-Kutta method (RK4). We also refer to the second order symmetric splitting method, described in the previous section, as SEJ. The symplectic method $\mathrm{MR}$ is based on a splitting of the the Hamiltonian $\mathcal{H}$ into four pieces.

$$
\tilde{\mathcal{H}}_{1}=\frac{\pi_{1}^{2}}{2 I_{1}}, \quad \tilde{\mathcal{H}}_{2}=\frac{\pi_{2}^{2}}{2 I_{2}}, \quad \tilde{\mathcal{H}}_{3}=\frac{\pi_{3}^{2}}{2 I_{3}}, \quad \tilde{\mathcal{H}}_{4}=u_{3} .
$$

Each of the corresponding Hamiltonian vector fields can be integrated exactly ( $\tilde{\mathcal{H}}_{1}, \tilde{\mathcal{H}}_{2}$, $\tilde{\mathcal{F}}_{3}$ correspond to the vector fields (8)), the symmetric composition of the flows gives rise to the approximation scheme,

$$
(\pi, u)^{(j+1)}=\Phi_{M}\left((\pi, u)^{(j)}\right)
$$

where

$$
\Phi_{M}=\varphi_{4, h / 2} \circ \Phi_{T, h} \circ \varphi_{4, h / 2}
$$

Here

$$
\Phi_{T, h}=\varphi_{1, h / 2} \circ \varphi_{2, h / 2} \circ \varphi_{3, h} \circ \varphi_{2, h / 2} \circ \varphi_{1, h / 2}
$$

is the contribution from the kinetic parts, $\tilde{\mathcal{H}}_{1}, \tilde{\mathcal{H}}_{2}$ and $\tilde{\mathcal{H}}_{3}$. The flows of kinetic parts correspond to elementary rotations in $\mathbf{R}^{3}$. E.g.

$$
\varphi_{1, h}\left((\pi, u)^{(j)}\right)=\left\{\begin{array}{l}
\pi^{(j+1)}=R_{x}(h) \pi^{(j)}, \\
u^{(j+1)}=R_{x}(h) u^{(j)}
\end{array}\right.
$$

where

$$
R_{x}(h)=\left(\begin{array}{ccc}
1 & 0 & 0 \\
0 & \cos \left(C^{(j)} h\right) & \sin \left(C^{(j)} h\right) \\
0 & -\sin \left(C^{(j)} h\right) & \cos \left(C^{(j)} h\right)
\end{array}\right)
$$

and

$$
C^{(j)}=\frac{(1)_{1}^{(j)}}{I_{1}}
$$

The flow for $\tilde{\mathcal{H}}_{4}$ is the same as for the system $S_{2}(10)$ of the previous section, i.e. $\varphi_{4, h}=\varphi_{h}^{\left|S_{2}\right|}$.

In the first experiment we consider the integration of the Euler equations. In Figure 1 we plot on the $x$-axis the execution time. taking the average over 200 experiments. required by the methods to perform the integration on the interval [0.1]. for different choices of the step size. On the y axis we report the corresponding values of the 2-nom of the global error. In all the experiments the reference solution for computing the global error is obtained using the built in function of MATLAB, Ode45, setting the absolute and relative tolerance equal to $10 \mathrm{c}-14$. We compare the MR method, which in this casc involves only the computation of the three flows corresponding to the Hamiltonians $\ddot{\eta}_{\text {. }}$ $\tilde{F}_{2}, \tilde{H}_{3}$, with the integration performed using the Jacobi elliptic functions. The latter method produces a very accurate solution of the problem (the error is of the sise of $10^{-14}$ ) and the error is independent on the step size of integration. In this experimemt dhe principal moments of inertial and the initial value for (1) are $l_{1}=1,1,-2,1 ;=3$ and ()$\left._{0}\right)=(1,0,2)^{r}$. respectively.

In the second experiment we perform the numerical integration of the Euler equations, with $I_{1}=1, I_{2}=2 . I_{3}=3$ and $\left(x_{11}=(1 .-2.1)^{l}\right.$. on the interval $\left.\mid(1) .+()\right) \mid$. In Figure 2 we consider the energy error ats the difference between the comstant exale 


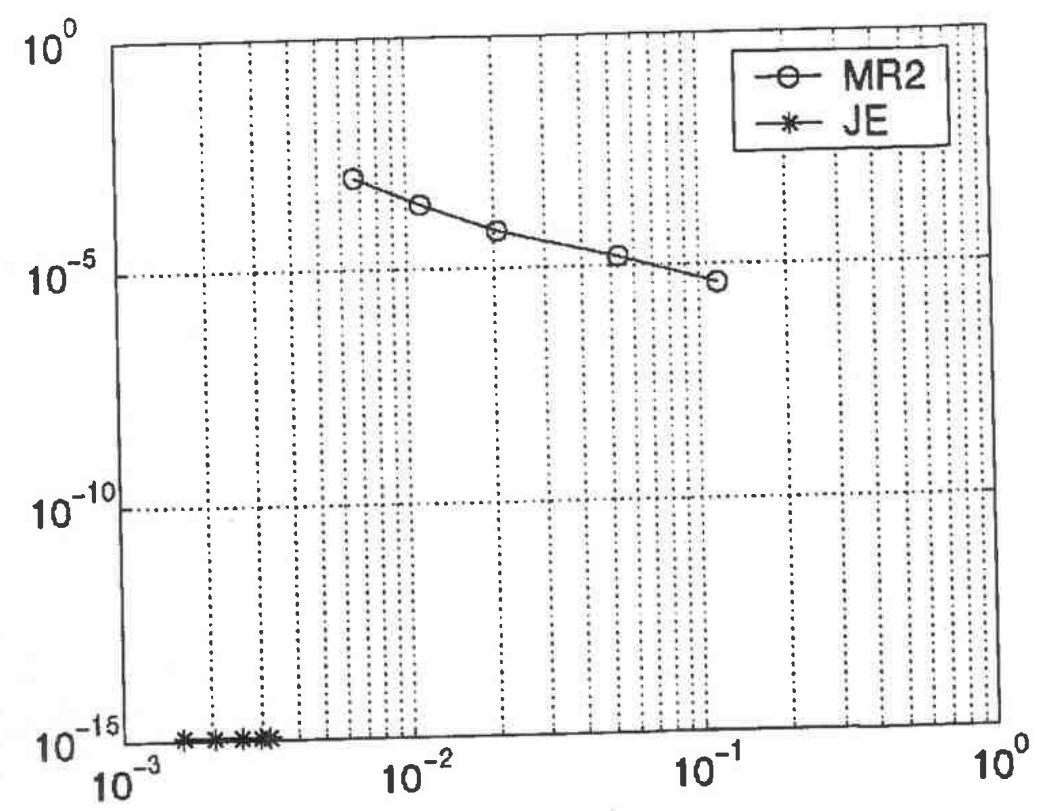

Figure 1. The Euler equations. Execution time, taking the average over 200 experiments, against the global error. Integration on the interval $[0,1]$ with different step sizes.

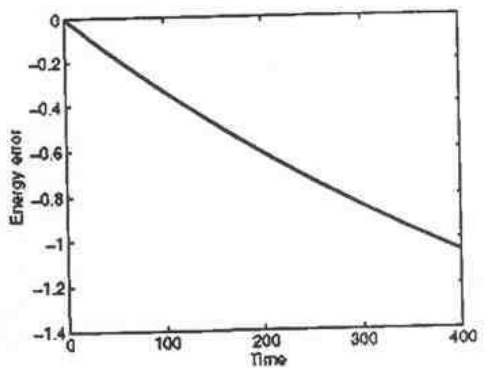

(a) RK4

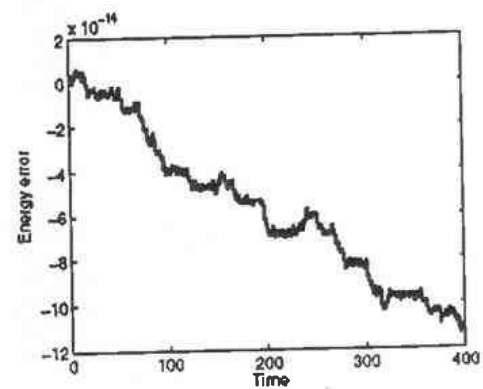

(c) Elliptic functions

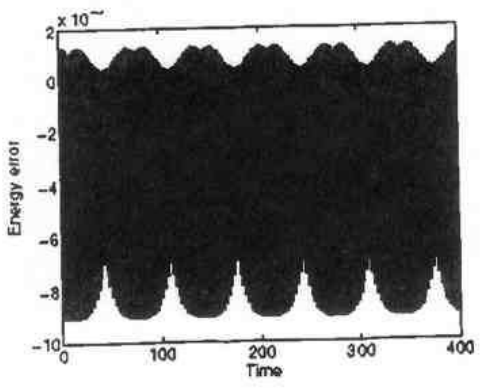

(b) MR

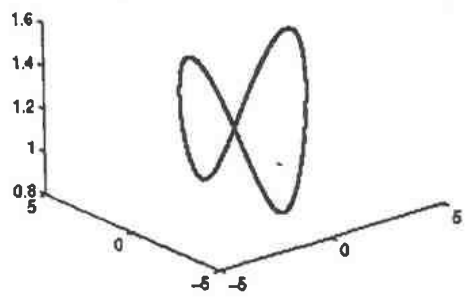

(d) Angular velocity, $\omega$

Figure 2. Energy for the free rigid body. Integration on the interval $[0,400]$. 


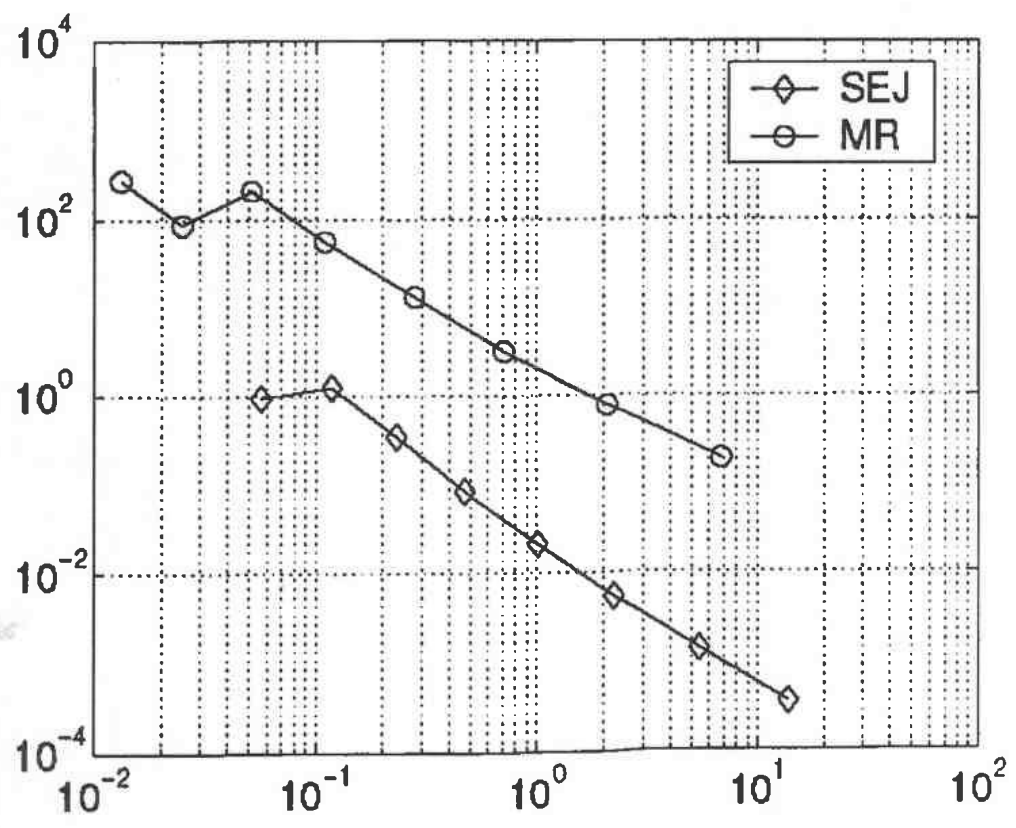

Figure 3. Heavy top. Execution time, taking the average over 100 experiments, against the global error. Integration on the interval $[0,1]$ with different step sizes.

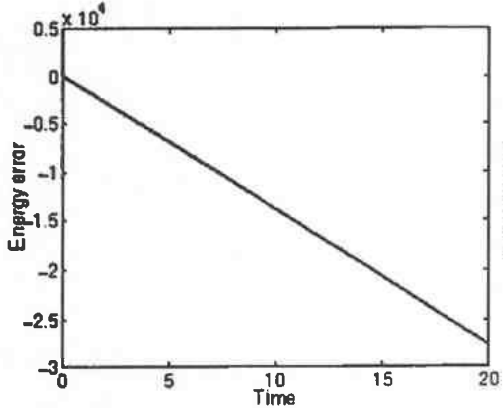

(a) RK4

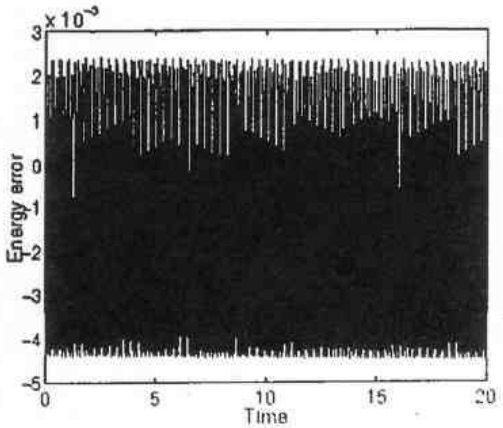

(c) SEJ

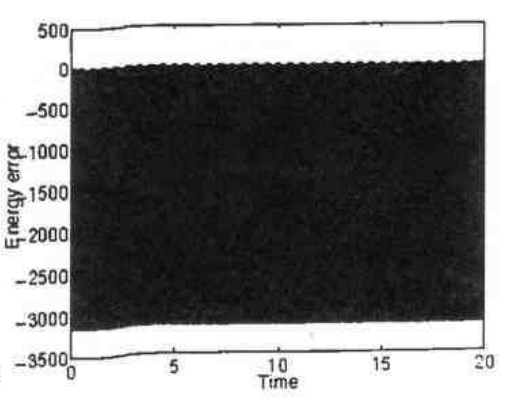

(b) MR

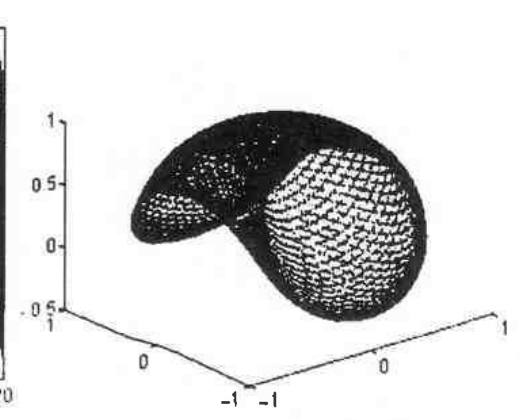

(d) Center of mass motion, $u$

Figure 4. Energy for the heavy top. Integration on the interval $[0,20]$, with step size $h=0.001$. 


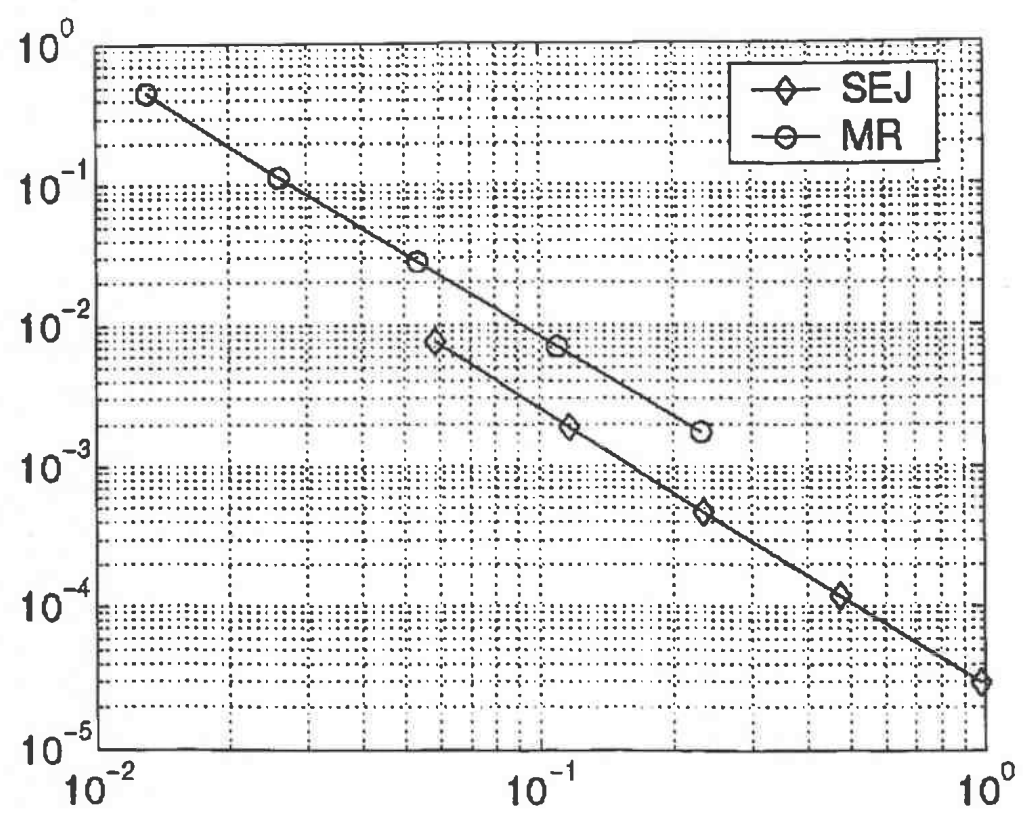

Figure 5. Heavy top. Execution time, taking the average over 200 experiments, against the global error. Integration on the interval $[0,1]$ with different step sizes.

energy, given by $\mathcal{H}$, and the energy obtained from the numerical methods (with step size $h=0.4$ ). We note that for the Runge-Kutta method there is a visible energy drift, for the MR method we observe a typical behavior of symplectic methods, i.e. the energy is oscillating around a constant value, (the amplitude of the oscillations is about $10^{-3}$ ), the method based on the use of the Jacobi elliptic functions computes a very accurate solution of the free rigid body problem and the energy is conserved to the same accuracy (the energy error is about $10^{-14}$ ). We conclude that computing the exact solution of the Euler equations, using ellipj of MATLAB, for computing the Jacobi elliptic functions, is a very efficient and accurate numerical approach for the Euler equations.

In Figure 3 we report the results of the third experiment. We consider the integration of the heavy top problem with the splitting methods MR and SEJ on the interval $[0,1]$, where the principal moments of inertia, the initial value for (1) and 1 are $I_{1}=1000$, $I_{2}=5000, I_{3}=6000,\left(1_{1}\right)=(100,100,10(0))^{T}$ and $I_{1}=(0,0,1)^{T}$, respectively. Also in this case we plot the execution time versus the global error for the two splitting methods. In this case the advantage of the new method is quite clear. In Figure 4 we repeat the experiment integrating on the interval $[0,20]$ with step size $h=0.001$. We report the energy error for the three numerical methods, RK4 (energy error $\left.=O\left(10^{4}\right)\right)$, MR and SEJ. The energy error has an oscillating behavior for both MR (energy error $=\mathcal{O}\left(10^{3}\right)$ ) and SEJ (energy error $=\mathcal{O}\left(10^{-3}\right)$ ).

In the next two experiments we consider the integration of a heavy top with the principal moments of inertia $I_{1}=1, I_{2}=5, I_{3}=6$. The initial values for (1) and $u$ are chosen ()$_{11}=(10,10,10)^{r}$ and $u_{10}=(0,0,1)^{r}$. We tirst integrate on the interval $|0,1|$ and compare the performance of the splitting methods in terms of exceution time against global error. Figure 5 . The two methods perform similarly, with a slightly advantage for the SEJ method.

Next we illustrate the qualitative performance of the two methods by comparing the results oblained by using different step sizes. We look at the energy error and the 


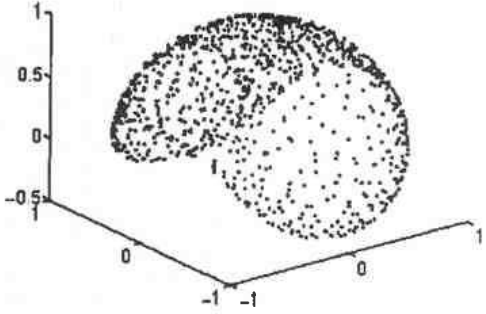

(a) Center of mass $u, h=0.1$, SEJ

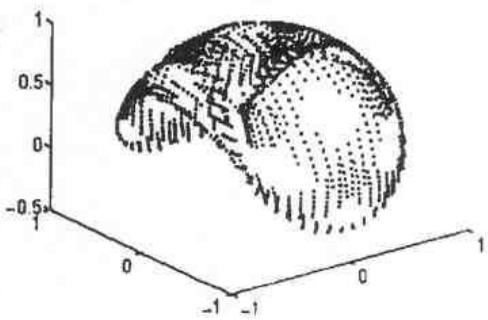

(c) Center of mass $u, h=0.05$, SEJ

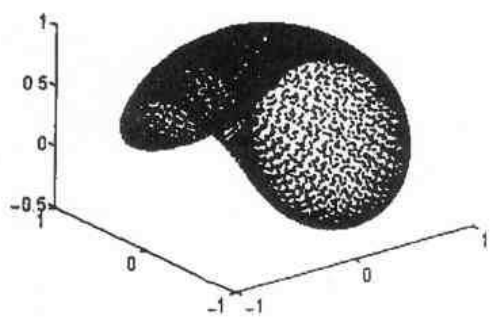

(e) Center of mass $u, h=0.01$, SEJ

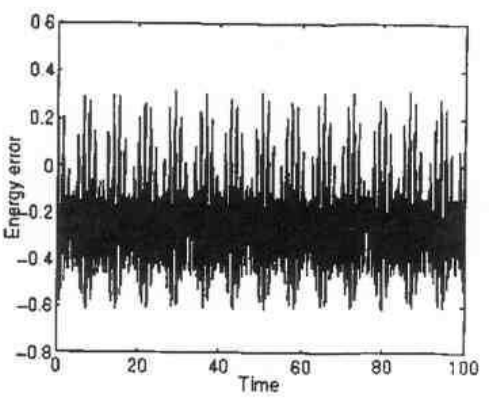

(b) SEJ, $h=0.1$

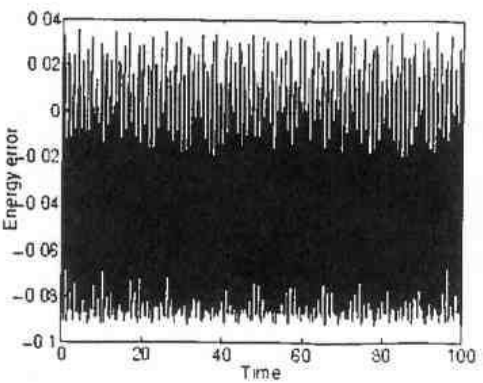

(d) SEJ, $h=0.05$

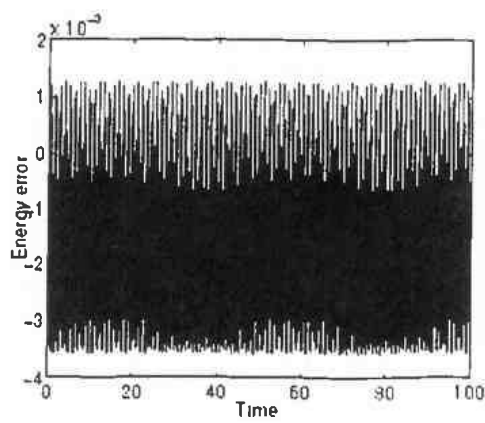

(f) $\mathrm{SEJ}, h=0.01$

Figure 6. Energy error for the heavy top. Integration on the interval [0, 100|, using the method SEJ. $I_{1}=1, I_{2}=5, I_{3}=6,(1)_{0}=(10,10,10)^{r}$.

numerical trajectory describing the motion of center of mass, in Figures 6 and 7. For step size $h=0.01$ the two methods produce similar trajectorics (the norm $\left.\left\|u_{\mathrm{SEJ}}-u_{\mathrm{MR}}\right\|=O\left(10^{-1}\right)\right)$. The energy error for SEJ is a factor $10^{-1}$ smaller than for MR. Increasing the step size to $h=0.05$ and $h=0.1$. the amplitude of the oscillations in the energy error increases for both methods. Consistently for all the experiments. SEJ has smaller energy error than MR. For $h=0.1$ the trajectory of the center of mass produced by the MR method becomes completely different. For the SEJ method the numerical trajectory of the center of mass preserves the same qualitative behavior for $h=(0.0)$. $h=0.05$ and $h=0.1$, Figure 6 .

Finally, we compare the energy error between the two methods. MR and SEJ. for different values of the inertia tensor. We consider the time interval $\mid 0.10)$ and integrate 


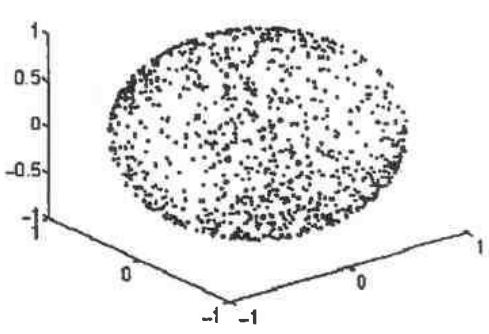

(a) Center of mass $u, h=0.1$, MR

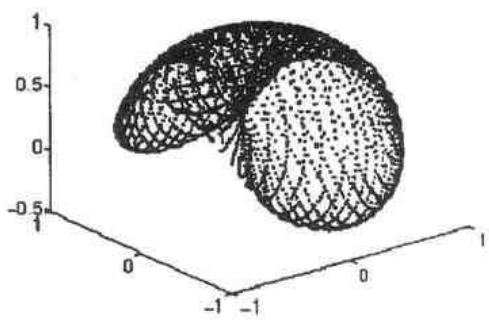

(c) Center of mass $u, h=0.05$, MR

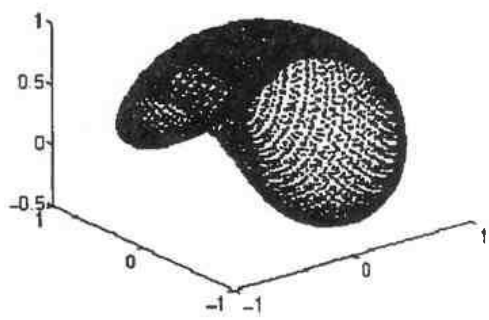

(e) Center of mass $u, h=0.01$, MR

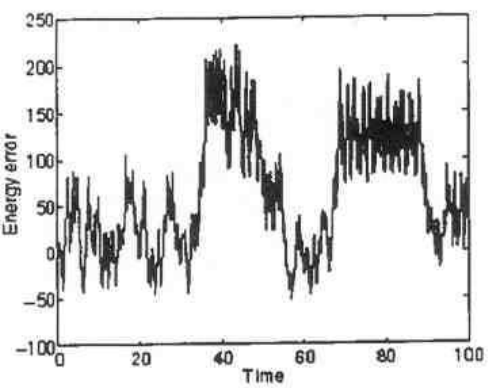

(b) $\mathrm{MR}_{3} h=0.1$

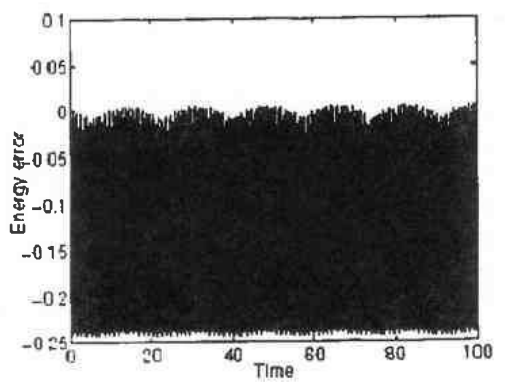

(d) $\mathrm{MR}, h=0.05$

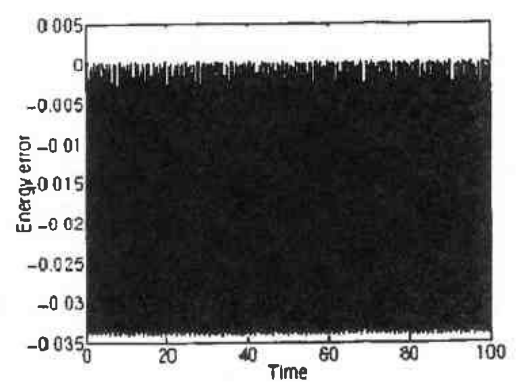

(f) $\mathrm{MR}, h=0.01$

Figure 7. Energy error for the heavy top. Integration on the interval $[0,100]$, using the method MR.

$$
I_{1}=1, I_{2}=5, I_{3}=6,(1)_{0}=(10,10,10)^{T} \text {. }
$$

with a fixed step size, $h=0.01$. In Figure 8 we report on the $y$-axis the average absolute value of the energy error,

$$
\frac{1}{N} \sum_{n=1}^{N}\left|\mathcal{H}-\mathcal{H}_{n}\right|
$$

with $\mathcal{H}_{n}$ the value of the numerical energy for the methods at time step $n$ and $\mathscr{H}$ the exact energy value, and $N=10 / 0.01=1000$. On the $x$-axis we report the value of a parameter $\alpha$ used for varying the principal moments of inertia, $I_{1}=\alpha, I_{2}=2 \alpha, I_{3}=3 \alpha$. The initial conditions for the angular velocity and the position of the center of mass are chosen $\left(_{1}=(1,2,3)\right.$ and $u_{0}=(0,0,1)$. Studying the graph 8 it appears that the energy error for the SEJ method is smaller compared to the MR method when the inertia is large, i.e. when the external torque is relative small compared to the momentum. 


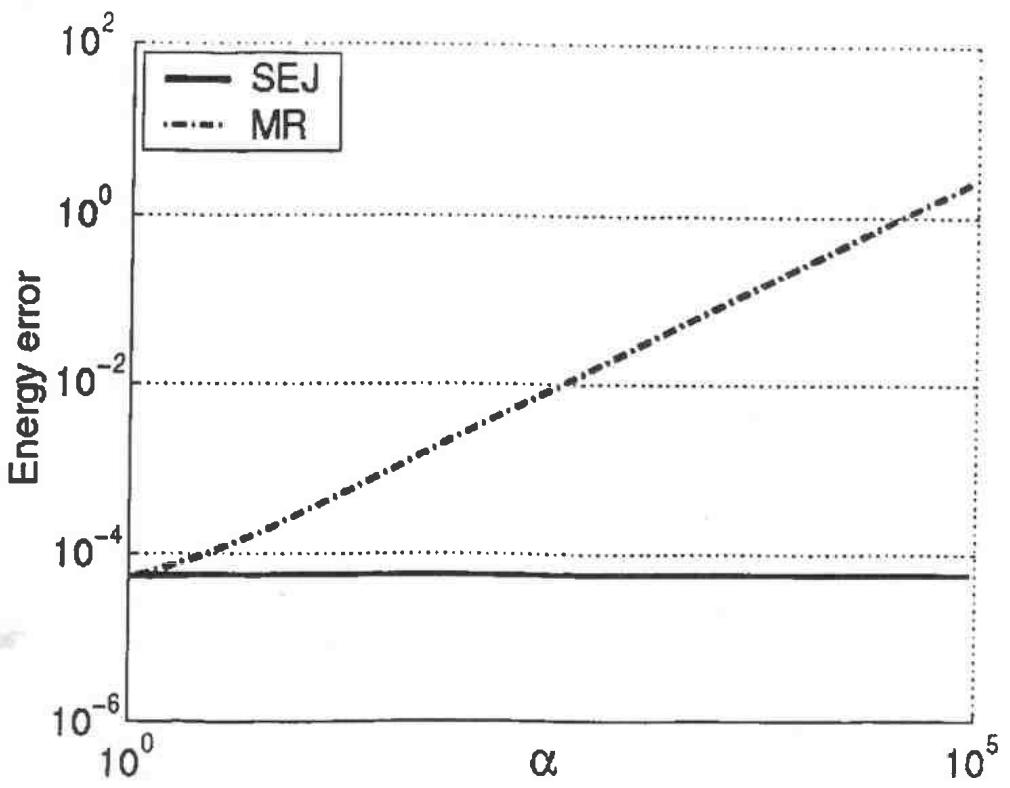

Figure 8. Energy error for the heavy top. Integration on the interval $[0,10], h=0.01$, using different inertia tensors, $I_{1}=\alpha, I_{2}=2 \alpha, I_{3}=3 \alpha, 1 \leq \alpha \leq 10^{5}$. The initial value for the center of mass, $u_{0}=(0$, $0,1)^{T}$, and angular velocity, $\left(\omega_{0}=(1,2,3)^{T}\right.$, are held fixt.

\section{Conclusions}

In this paper we presented a symmetric splitting method for the integration of rigid body problems subject to external forces. The numerical strategy is based on the use of available efficient algorithms for the computation of Jacobi elliptic functions. We show that thanks to its symmetry, the method performs much better then classical integrators. like the Runge-Kutta method of order 4 , in the long time integration of the considered problems. We also compared the method with a similar symplectic splitting method which inspired the present approach. In many of the performed experiments the presented symmetric splitting is more efficient then the symplectic splitting. giving smaller global error for the same amount of floating point operations. Moreover the new method presents in our experiments al better energy conservation. This seems to be true especially for problems where the principal moments of inertia are large.

\section{References}

ABRAMOWITZ, M. \& STIiciun. I. A. (1992). Handhook of mathematical functions with formulas, graphs, and mathematical tables, volume 55 of National Bureau of Standards Applied Mathematics Series. 55. Reprint of the 1972 edition. Dover Publications, Inc., New York.

Butcher, J. (2003). Numerical Methods for Ordinary Differential Equations. Wiley, second edition edition.

Celledoni. E. \& Owrin, B. (20)(03). Lie group methods for rigid body dynamics and time integration on manifolds. Comput. Methods Appl. Mech. Engrg., 192, 421-438.

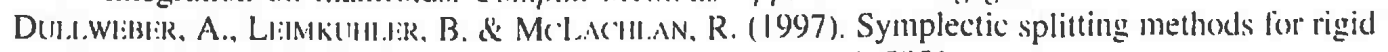
body molecular dynamics. J. Chem. Phis.s. 107, 5840-5851.

Ge, Z. \& MArsil:N, J. E. (1988). Lic-Poision Hamilton-Jacobi theory and Lie-Poisson integrators. Physicis Letlers A. 13.3(3). 1.34-1.39.

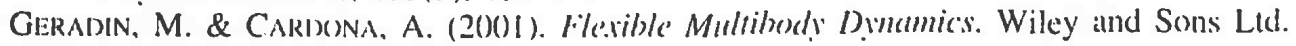

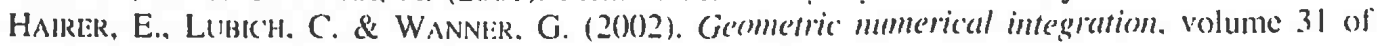
Springe'r series in compuitational mathe'matic's. Springer. 
LeIMKuhler, B. \& REICH, S. (2004). Simulating Hamilhomian Dymamic's, volume 14 of Cambridge Monographs on Applied and Computational Mathematics. Cambridge University Press, first edition edition.

LEwIS, D. \& SIMO, J. C. (1994). Conserving algorithms for the dynamics of Hamiltonian systems of Lie groups. J. Nonlinear Sci., 4, 253-299.

Marsden. J. E. \& Ratiu, T. S. (1994). Introduction to Mechanics and Symmetry. Springer-Verlag.

MCLaChlan, R. I. (1993). Explicit Lie-Poisson integration and the Euler equations. Physical Review Letters, 71, 3043-3046, 1993.

MCLACHLAN, R. I. \& ZANNA, A. (2005). The discrete Moser-Veselov algorithm for the free rigid body, revisited. Found. of Comp. Math., 5(1), 87-123.

Mitchell, J.WM. (2000). A simplified variation of parameters solution for the motion of an arbirarily torqued mass asymmetric rigid body. PhD thesis, University of Cincinnati.

Moser, J. \& Veselov, A. (199i). Discrete versions of some classical integrable systems and factorization of matrix polynomials. J. of Comm. Math. Phys., 139(2), 217-243.

REICH, S. (1996). Symplectic integrators for systems of rigid bodies. Integration algorithms and classical mechanics (Toronto, ON, 1993). Fields Inst. Commun., 10, 181-191. 